\title{
Compliant Mechanisms in Progress and Development Of Modern Technology
}

\author{
D. LATEȘ1,2, M. CĂȘVEAN 3
}

1University of Medicine, Pharmacy, Science and Technology of Târgu Mures, E-mail: lates.upm@gmail.com 2iFOR Research and development center of IRUM, Târgu Mureș, E-mail: daniel.lates@irum.ro

${ }^{3}$ Accesa IT Systems, Cluj-Napoca, Cluj, E-mail: marius.casvean@yahoo.com

Abstract. In this paper, the compliant mechanisms will be presented. There will be presented some representative works that have brought progress and development in modern technology. Examples will be presented from simple concepts such as a tweezers, staples, to mechanical clutches, pointer and micro-compliant mechanical structures.

\section{Introduction}

There are many types of mechanisms nowadays. A mechanism is a device to transfer or transform motion, force or energy. Traditional rigid body mechanisms consist of rigid links connected at movable joints. Whatever machines you see in your day to day life has some underlying mechanisms that govern its motion to produce the desired output. The energy is transfered from the input to the output. Compliant mechanisms have been used for decades in different fields and industries. In particular, robotics, medical or aerospace have been using such mechanisms for some 50 years now, even more. These mechanisms depend on some parameters, like elastic deformations, to provide precise and smooth motions. It is essential to develop an accurate model to quatify the deformation for kinetic analysis and parameter optimization.

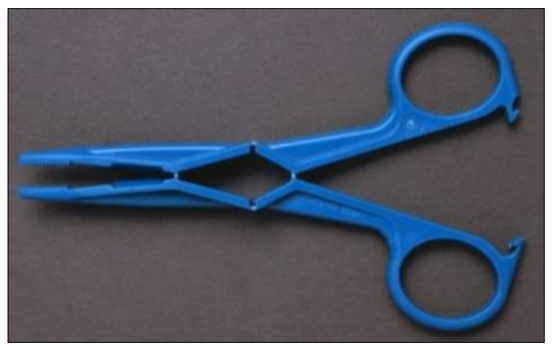

Figure 1. Compliant tweezers.

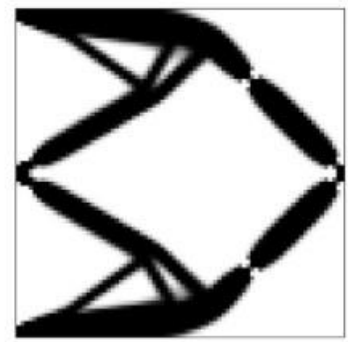

Figure 2. Displacement inverter mechanism.

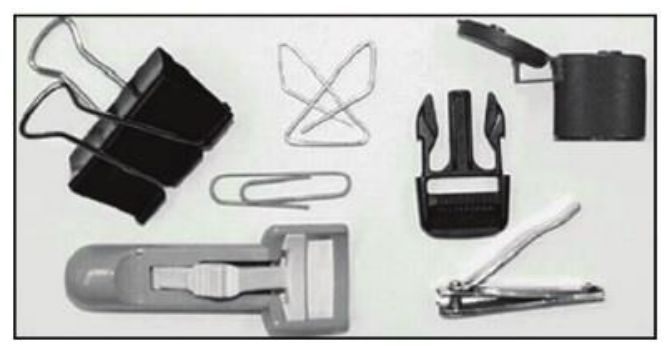

Figure 3. Common compliant devices.

Compliant mechanisms are monolithic structures where the movement is given by the flexibility of the structure rather than the presence of joints and pins. The absence of joints allows the construction of compliant mechanisms in microscale. This kind of mechanism transfer motion, force or energy. Unlike rigid-link mechanisms, compliant mechanism gain at least some of their mobility from the deflection of flexible members rather than from movable joints only. Basically not all the links of the mechanism 
need to be flexible for it to be named as a compliant system but some important links must be flexible. Fully compliant mechanisms are very unstable and unreliable.
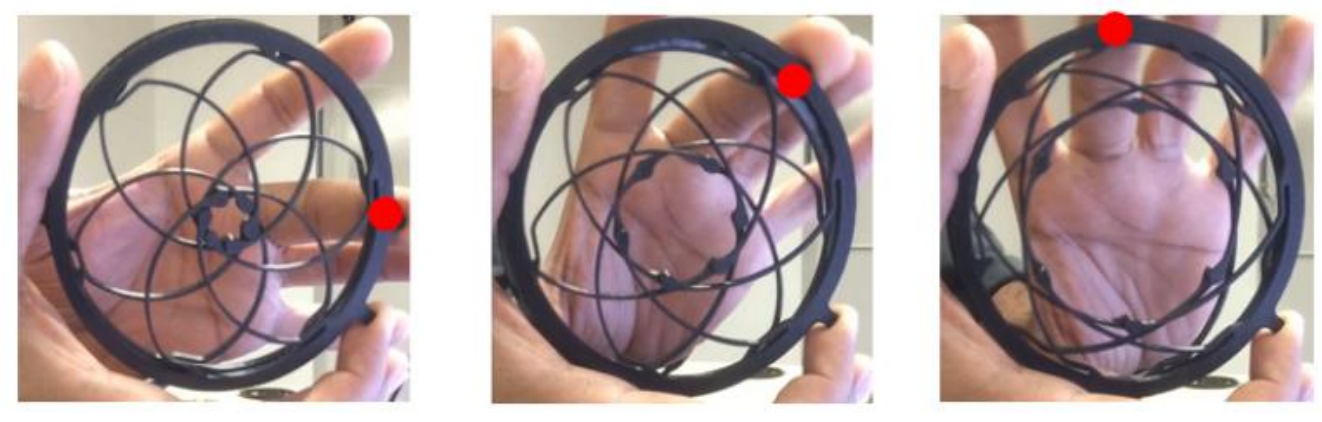

Figure 4. Compliant torsional spring.

Also, these mechanisms rely upon elastic deformation to perform their function of transmitting and transforming motion and force. From an overall perspectve that consider performance, economy of material, scalability to micro and nano sizes, etc., compliant mechanisms are preferable over rigid body mechanisms.

\section{International papers}

A large number of papers exists, regarding the compliant mechanisms. It is not the concept that can be treated as something new, but it is the role that can be assigned to it. And the shape may looks always simple, but there is invested a large amount of time to investigate how this should look like for doing the proper desired functionality. Optimizations are welcome anytime.

For a continuum topology design problem, the optimal solution is a structure that is composed of only one material and voids, which means that a $0-1$ decision is used to determine whether the material should be placed at a point within the design domain.

Other papers can bring a short overview of some mechanical structures and their performance characteristics. One of them presents three concepts of force amplifiers and results from simulations are discussed. The specific design of the linear motion force amplifier/motion reduction for a high accuracy positioning device with large payload capacity is studied.

\section{Representative papers with impact in modern industry}

Compliant mechanisms have made an enormous contribution in various fields. Several methods have being conceived to analyze and design these compliant mechanisms that gain part of their motion from deflection of flexible members rather than movable joints, as conventional mechanisms. Recently, many familiar example of compliant mechanisms have been designed and widely used in various field as automotive industry, aerospace industry, MEMS, Medical devices, Robotic arm with minimal impedance and assistive mechanisms. For adaptive structures, components in transportation, etc., the largest challenge is to analyze and design these mechanisms. 
Consider the compliant over-running clutch and its rigid body counterpart. Considerably fewer components are required for the compliant mechanism than the rigid mechanism. The reduction in part count may reduce manufacturing and assembly time and cost. Compliant over-running clutch is an example for application of compliant systems in automotive industry, apart from that the car wiper, steering parts, gears, breaks are other examples. The compliant mechanisms and systems are also used in (new age) industry like aerospace, mechanism amplification for sensors and actuators or for microsurgery suturing device, etc.

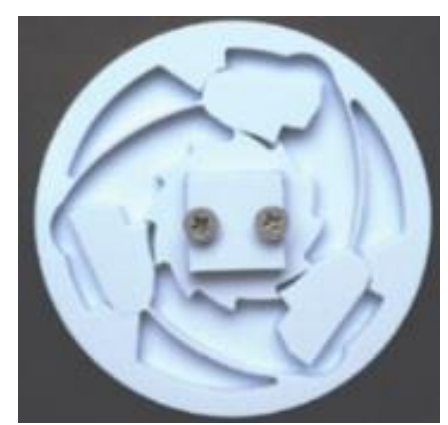

Figure 5. Over-running clutch

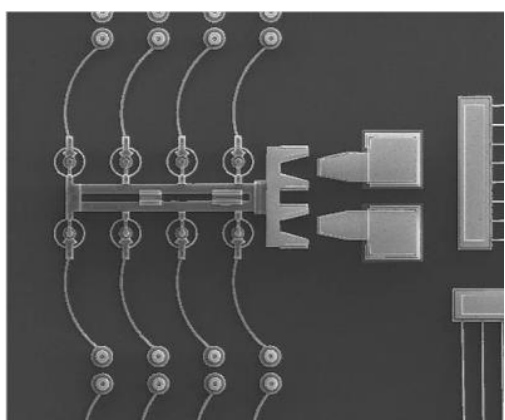

Figure 6. Micro mechanisms

Morphing aircraft structures can significantly enhance air vehicle performance. The aerodynamic benefits are exploit. Computational tools are being developed to design structures that deform into specified shapes given simple actuators inputs. These synthesis methods seeks to optimize the stiffness of the structure to minimize actuator effort and maximize the stiffness with respect to the environment.

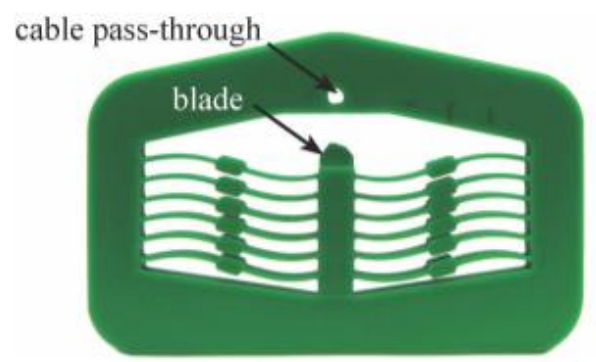

Figure 7. Cutter non-explosive release mechanism

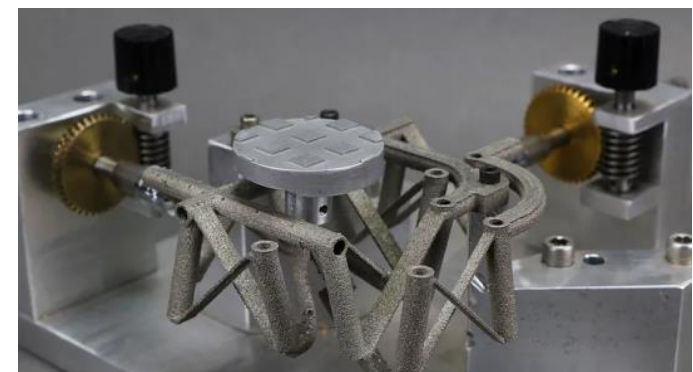

Figure 8. The pointer device

Mechanisms that possess distributed compliance, as opposed to lumped compliance, are much more fatigue resistant and easier to manufacture. Several computational approaches have been developed to design compliant mechanism for desired force-displacement characteristics and more recently for generalized shape change.

Compliant mechanisms offer undisputable advantages, in term of reliability and performance and as such can be a great ally in the search for enhanced quality. The potential for dramatic reduction in the total number of parts required to accomplish a specific task. 


\section{Acknowledgments}

We would like to express our gratitude to SC IRUM SA for the moral and financial support for the realization of this article. We also want to thank the organizers of the event for organizing such a scientific conference that helps us publish articles on engineering.

\section{References}

[1] Muqing Niu, Bintang Yang, Yikun Yang, Guang Meng,(2018),Two generalized models for planar compliant mechanisms based on tree structure method,Precision Engineering, vol.51, pag.137-144

[2] Cicero R. de Lima, Glaucio H. Paulino, (2019), Auxetic structure design using compliant mechanisms: A topology optimization approach with polygonal finite elements, Advances in Engineering Software, vol. 129, pag. 69-80.

[3] M. P. Bendsoe, O. Sigmund, (2004), Topology Optimization: Theory. Methods and Applications, Springer.

[4] Jaroslav Hricko, Stefan Havlik, Compliant mechanisms for motion/force amplifiers for robotics, Advances in Intelligent Systems and Computing (AISC), vol. 980.

[5] Pandiyan Arumugam, Arun Kumar, (2016), Design methods for compliant mechanisms used in new age industry - a review, Istrazivanja i projektovanja za privredu, vol. 14, pag. 223-232.

[6] Sridhar Kota, Joel Hetrick, Russell Osborn, Donald Paul, Ed Pendleton, Peter Flick, Carl Tilmann, (2003), Design and application of compliant mechanisms for morphing aircraft structures, Proceedings of SPIE - The International Society for Optical Engineering.

[7] Lateș, D., (2014), Cercetări teoretice și aplicative privind mecanismele compliante folosite în mecatronică, Teza de Doctorat, Cluj-Napoca.

[8] J. Agnus, N. Chaillet, C. Cl'evy, S. Demb'el'e, M. Gauthier, Y. Haddab, G. Laurent, P. Lutz, N. Piat and M. Rakotondrabe, (2013), Robotic Microassembly and micromanipulation at FEMTO-ST (https://gjlaurent.github.io/assets/papers/Agnus2013robotic.pdf).

[9] Shannon A. Zirbel, Kyler A. Tolman, Brian P. Trease, Larry L. Howell, (2016), Bistable Mechanisms for Space Applications, PLOS ONE | DOI:10.1371/Journal.Pone.0168218

[10] https://monochrome-watches.com/technical-perspective-flexure-revolution-compliantmechanisms -applied- watchmaking/

[11] https://ieee.nitk.ac.in/blog/compliant-mechanisms/

[12] http://www.um.u-tokyo.ac.jp/people/faculty_matsumoto_en.html (Microcell for optical components at University of Tokyo, Matsumoto 2007)

[13] https://www.compliantmechanisms.byu.edu/about-compliant-mechanisms

[14] https://www.goengineer.com/2018/01/26/the-challenge-of-tuning-compliant-torsionalsprings/3d-printing/ 